\title{
Effect of salt stress on the growth and photosystem II photochemical characteristics of Lycium ruthenicum Murr. seedlings
}

\author{
Y.-Y. GUO*, H.-S. NIE ${ }^{* *}$, H.-Y. YU ${ }^{*}$, D.-S. KONG ${ }^{*}$, and J.-Y. WU* \\ Key Laboratory of Hexi Corridor Resources Utilization of Gansu, Hexi University, Zhangye, Gansu 734000, \\ China* \\ Graduate School of Agriculture 3-5-8 Saiwai-cho, Tokyo University of Agriculture and Technology, Tokyo 183- \\ 8509, Japan**
}

\begin{abstract}
The present study aimed to determine effects of salt stress on Lycium ruthenicum Murr. seedlings. Our results showed that mild and moderate salt stress were beneficial to L. ruthenicum seedling growth. Minimal fluorescence increased and maximum fluorescence decreased gradually with the increasing levels of salt stress. Absorption flux per reaction center (RC), trapped energy flux per RC, and trapped energy flux per optical cross section (CS) increased significantly, while electron transport flux per CS decreased with salt stress duration and rising salt concentration. During salt stress, there was a gradual decline in probability that a trapped exciton moves an electron into the trapped electron transport chain beyond $\mathrm{Q}_{\mathrm{A}}$, quantum yield for electron transport, and performance index on absorption basis. However, gradual increases in relative variable fluorescence, dissipated energy flux per RC, and dissipated energy flux per CS were found in response to salt stress.
\end{abstract}

Additional key words: chlorophyll fluorescence kinetics; OJIP transient; photosynthesis; salt tolerance.

\section{Introduction}

During their life time, plants may face various abiotic stresses, such as salt excess, drought, and temperature fluctuations, all of which may negatively affect their full growth potential (Forni et al. 2017, Yousuf et al. 2017, George et al. 2018). Salt stress is the most serious abiotic stress that threatens agricultural sustainability by negative impact on plant growth and crop production (Bray et al. 2000, Azeem et al. 2017, Yousuf et al. 2017). The accumulation of salts in soil leads to water stress and nutrient deficiency in plants (Arshi et al. 2012). Salt stress may lead to redox imbalances, ion toxicity, oxidative damage, and water deficit by destroying ion and osmotic homeostasis, which can lead to the inhibition of photosynthesis, metabolic dysfunction, and damage to cellular structures within plant cells (Wei et al. 2017). Salt stress can affect light reaction, such as chlorophyll photoenergy conversion and electron transport, and dark reaction in plants (Sudhir and Murthy 2004, Zhang et al. 2009, Ashraf and Harris 2013, Zhang et al. 2013, Zhou et al. 2016). Therefore, the response and adaptation of plant growth, biomass allocation, and chlorophyll characteristics to salt stress is crucial in further discussing plant adaptation to salt.

Lycium ruthenicum Murr., which belongs to Solanaceae family, is a perennial deciduous shrub tolerant to drought and salt. This plant, which is mainly distributed in Northwest China, is important in ecology, medicine, forestry, and particularly in water and soil conservation. Rich in protein and polysaccharides, the L. ruthenicum fruit has been recorded in Tibetan medical classic 'Jing Zhu Ben Cao' as a traditional herb (Zheng et al. 2011) and has become increasingly expensive due to the acceptance of its medicinal value. Owing to a serious damage to natural vegetation, L. ruthenicum grew fewer in number, and the species was listed as an important conservation plant. Thus, effective management and protection of

\footnotetext{
$\overline{\text { Received }} 11$ January 2018, accepted 22 October 2018.

${ }^{+}$Corresponding author; phone: 86-13993693097, e-mail: yhy20001113@163.com

Abbreviations: $\mathrm{ABS} / \mathrm{CS}_{0}$ - absorption flux per CS; ABS/RC - absorption flux per RC; CK - control; CS - optical cross-section; DAE days of experiment; $\mathrm{DI}_{0} / \mathrm{CS}_{0}$ - dissipated energy flux per $\mathrm{CS} ; \mathrm{DI}_{0} / \mathrm{RC}$ - dissipated energy flux per $\mathrm{RC} ; \mathrm{ET}_{0} / \mathrm{CS}_{0}$ - electron transport flux per $\mathrm{CS} ; \mathrm{ET}_{0} / \mathrm{RC}$ - electron transport flux per $\mathrm{RC} ; \mathrm{F}_{0}-$ minimal fluorescence; $\mathrm{F}_{\mathrm{m}}$ - maximum fluorescence; $\mathrm{F}_{\mathrm{v}} / \mathrm{F}_{\mathrm{m}}-$ maximum quantum yield of PSII; OJIP - fast chlorophyll fluorescence transients; $\mathrm{PI}_{\text {abs }}$ - performance index on absorption basis; $\mathrm{RC}$ - reaction center; $\mathrm{RC} / \mathrm{CS}_{0}$ - amount of active PSII RC per CS; ST1 - 0.1 mol(salt) $\mathrm{L}^{-1} ; \mathrm{ST} 2-0.3 \operatorname{mol}(\mathrm{salt}) \mathrm{L}^{-1}$; ST3 - 0.5 mol(salt) $\mathrm{L}^{-1}$; R:S - the root to shoot ratio; $\mathrm{TR}_{0} / \mathrm{CS}_{0}$ - trapped energy flux per $\mathrm{CS} ; \mathrm{TR}_{0} / \mathrm{RC}$ - trapped energy flux per $\mathrm{RC} ; \mathrm{V}_{\mathrm{j}}$ - relative variable fluorescence; $\varphi_{\mathrm{DO}}$ - quantum yield at $\mathrm{t}=0$ for energy dissipation; $\varphi_{\mathrm{EO}}$ - quantum yield for electron transport; $\varphi_{\mathrm{PO}}$ - maximum quantum yield of primary photochemistry; $\psi_{\mathrm{o}}$ - probability that a trapped exciton moves an electron into the trapped electron transport chain beyond $\mathrm{Q}_{\mathrm{A}}$. Acknowledgements: This work was supported by programs of National Natural Science Foundation $(31460189,31660193)$ and the Scientific Research Project of the Higher Education Institutions of Gansu Province (2014A-111).
} 
L. ruthenicum should be developed. Field investigation results indicated a reduced seedling number; whether salt stress affects a seedling number required further research. Several studies suggested that seed germination was affected by drought and salt (He et al. 2011, Han et al. 2014). However, information on seedling adaptive capacity and mechanism of plant adaptation under salt environments is still lacking.

Thus, the present study aimed to determine the effect of salt stress on the biomass allocation and chlorophyll (Chl) fluorescence of L. ruthenicum seedlings. Comparison of these responses benefits the determination of salt tolerance capacity and mechanism in L. ruthenicum cultivars and provides theoretical basis for the vegetation restoration of L. ruthenicum.

\section{Materials and methods}

Plant material and salt stress: The study site was located in Hexi University Practice Base of Agriculture, Zhangye City $\left(37^{\circ} 28^{\prime} \mathrm{N}, 97^{\circ} 20^{\prime} \mathrm{E}\right)$ in Gansu Province, China. The climate was a continental environment with an annual average temperature, sunshine duration, rainfall, and evaporation of $6^{\circ} \mathrm{C} ; 3,106 \mathrm{~h} ; 113-120 \mathrm{~mm}$; and 2,291 $\mathrm{mm}$, respectively. Seeds were harvested in August 2015 at Ganzhou District, Gansu Province, China. After washing, seeds were dried for one week under direct sunlight and then stored at $4^{\circ} \mathrm{C}$ prior to experiments. After the seeds were surface-sterilized with $2 \%$ potassium permanganate solution, five seeds were sowed in each pot $(23 \mathrm{~cm}$ in diameter and $20 \mathrm{~cm}$ in height) on 15 March, 2016, with the pots containing the same amount of soil, sandy soil, and humus $(1: 2: 1)$. When the seedling formed $2-3$ leaves, three seedlings were retained in each pot. Stress treatment was initiated on 10 May, 2017. The experiment consisted of control and salt-treated groups, with ten pots assigned to each group. The control and stressed groups were subjected to normal watering. After experiment, the stress treatment was applied to three groups. The salt-treated group was watered once a week, and $0.1 \mathrm{~mol} \mathrm{~L}^{-1}$ (ST1), $0.3 \mathrm{~mol} \mathrm{~L}^{-1}$ (ST2), and $0.5 \mathrm{~mol} \mathrm{~L}^{-1}$ (ST3) $\mathrm{NaCl}$ salt solutions were used. At days $0,7,14$, and 21 (DAE), growth parameters, biomass, and fluorescence parameters were measured in control and stress-treated plants.

Growth parameters: At 0, 7, 14, and 21 DAE, height, base diameter, branching number, and root length were measured in control and stressed plants. Plant height and root length were measured using a tape. The seedlings were harvested, divided into roots, stems, and leaves, and dried in an oven for $48 \mathrm{~h}$ at $70^{\circ} \mathrm{C}$ for biomass determination. The total biomass was the sum of root, stem, and leaf dry mass. Root:shoot (R:S) ratio was calculated as the root dry mass (DM) divided by the aboveground DM.

Fast Chl fluorescence induction curve: The fluorescence parameters were determined on the attached leaf by a plant efficiency analyzer (Handy-PEA, Hansatech Instrument Ltd., UK) with an actinic light of 3,000 $\mu$ mol(photon) $\mathrm{m}^{-2} \mathrm{~s}^{-1}$ using the procedure described by Strasser et al.
(1995). The selected leaves of control and stressed plants were placed in darkness for $20 \mathrm{~min}$ before the fluorescence parameters were measured at $0,7,14$, and 21 DAE. On the basis of the theory of energy fluxes in PSII, we obtained several fluorescence parameters from the OJIP transient curve via the following original values of fluorescence intensity at $50 \mu \mathrm{s}\left(\mathrm{F}_{0}\right)$ and the maximum fluorescence intensity $\left(\mathrm{F}_{\mathrm{m}}\right)$. Several parameters selected to quantify the PSII behavior of L. ruthenicum Murr. were calculated from the above original data (Strasser et al. 1995).

Statistical analysis was performed using SPSS18.0 statistical software package for Windows. All data were subjected to one-way analysis of variance (ANOVA). LSD multiple comparison tests were used to separate significant differences between all treatments at 0.05 level. Standard error (SE) was calculated, as shown in figures and tables.

\section{Results}

Growth: In all treatments, height and base diameter were significantly higher in ST1 than those in other treatments (Fig. 1 $A, B$ ). With increasing stress duration, the height, base diameter, branching number, and crown increased in all treatments. However, ST1 and ST2 presented higher growth parameters compared to other treatments. In CK, ST1, ST2, and ST3, height increased by 3.0, 9.9, 4.9, and $2.7 \%$ at 7 DAE compared with 0 DAE, respectively. At 21 DAE, height increased by $15.2,34.9,21.0$, and $11.0 \%$ compared to those of $0 \mathrm{DAE}$, while the base diameter increased by $88.6,167.6,100.5$, and $56.2 \%$ compared to those at 0 DAE in CK, ST1, ST2, and ST3 treatments, respectively. The branching number increased by 12.9 , $40.0,15.6$, and $12.5 \%$, and the crown increased by 41.4 , $83.5,61.7$, and $35.5 \%$ at $21 \mathrm{DAE}$ compared to that at 0 DAE in CK, ST1, ST2, and ST3, respectively (Fig. 1C,D).

Biomass: The dry mass allocation of stress-treated seedlings was significantly different from that of the control treatment (Table 1). With increasing treatment duration, the total DM, leaf mass, stem mass, and root mass of seedlings increased across all treatments as follows: ST1 $>\mathrm{ST} 2>\mathrm{CK}>\mathrm{ST} 3$. At $21 \mathrm{DAE}$, the total DM of ST1 seedling increased by $29.6,22.6$, and $47.4 \%$ compared to those in CK, ST2, and ST3, respectively. The R:S ratios in ST1 and ST2 were higher at 21 DAE compared to those in other treatments, but those in CK and ST3 were higher at 14 DAE. Root length increased with treatment duration in all treatments. However, at the same treatment time, the root length exhibited the following relation for different treatments: ST1 > ST2 > CK > ST3 at 14 and 21 DAE. At 21 DAE, root length of CK, ST1, ST2, and ST3 seedling increased by $62.1,119.1,68.1$, and $46.2 \%$ compared to those at 0 DAE

Chl fluorescence transients: After L. ruthenicum seedling leaves were exposed to pulse light intensity of saturation, the Chl fluorescence value increased rapidly through dark adaptation. However, the value eventually decreased (Fig. 2). The OJIP curves showed little change at different 


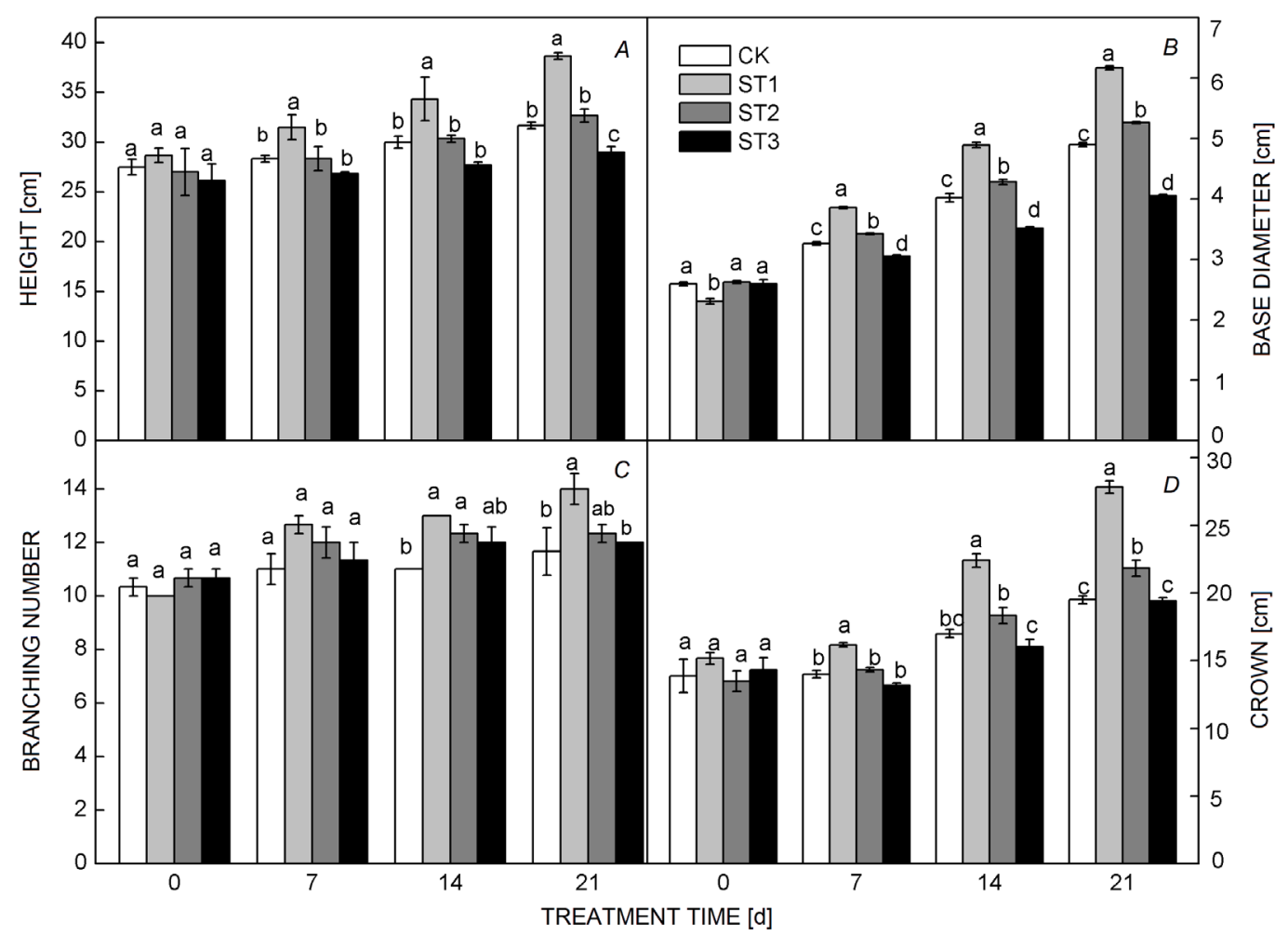

Fig. 1. Seedling height $(A)$, base diameter $(B)$, branch number $(C)$, and crown $(D)$ of control and salt-treated Lycium ruthenicum Murr. seedlings. Data are means $\pm \mathrm{SE}(n=5)$. Different letters indicate significant differences between treatments at $P<0.05$. CK - control; salt treatments: ST1 $-0.1 \mathrm{~mol} \mathrm{~L}^{-1}, \mathrm{ST} 2-0.3 \mathrm{~mol} \mathrm{~L}^{-1}$, ST $3-0.5 \mathrm{~mol} \mathrm{~L}^{-1}$.

Table 1. Seedling biomass, root mass, root to shoot ratio (R:S), and root length of control and salt-treated Lycium ruthenicum Murr. seedlings. Data are means \pm SE $(n=5)$. Different letters indicate significant differences between treatments at $P<0.05$. CK - control, DAE - days of experiment, DM - dry mass, salt treatments: ST1 $-0.1 \mathrm{~mol} \mathrm{~L}^{-1}$, ST2 - $0.3 \mathrm{~mol} \mathrm{~L}{ }^{-1}$, ST $3-0.5 \mathrm{~mol} \mathrm{~L}^{-1}$.

\begin{tabular}{|c|c|c|c|c|c|c|c|}
\hline Treatment & DAE & Total DM [g] & Leaf DM [g] & Stem DM [g] & Root DM [g] & $\mathrm{R}: \mathrm{S}$ & Root length $[\mathrm{cm}]$ \\
\hline \multirow[t]{4}{*}{ CK } & 0 & $1.29 \pm 0.02^{\mathrm{a}}$ & $0.74 \pm 0.01^{\mathrm{a}}$ & $0.34 \pm 0.01^{\mathrm{a}}$ & $0.21 \pm 0.01^{\mathrm{a}}$ & $0.20 \pm 0.01^{\mathrm{a}}$ & $14.50 \pm 0.29^{\mathrm{a}}$ \\
\hline & 7 & $1.63 \pm 0.01^{\mathrm{b}}$ & $0.84 \pm 0.00^{\mathrm{b}}$ & $0.47 \pm 0.00^{\mathrm{b}}$ & $0.32 \pm 0.00^{\mathrm{b}}$ & $0.25 \pm 0.00^{\mathrm{b}}$ & $17.50 \pm 0.29^{b}$ \\
\hline & 14 & $2.16 \pm 0.04^{c}$ & $1.12 \pm 0.03^{\mathrm{c}}$ & $0.61 \pm 0.00^{c}$ & $0.44 \pm 0.01^{\mathrm{c}}$ & $0.26 \pm 0.01^{\mathrm{c}}$ & $20.50 \pm 0.29^{c}$ \\
\hline & 21 & $3.07 \pm 0.01^{\mathrm{d}}$ & $1.73 \pm 0.01^{\mathrm{d}}$ & $0.82 \pm 0.00^{\mathrm{d}}$ & $0.52 \pm 0.00^{\mathrm{d}}$ & $0.20 \pm 0.00^{\mathrm{a}}$ & $23.50 \pm 0.29^{d}$ \\
\hline \multirow[t]{4}{*}{ ST1 } & 0 & $1.32 \pm 0.01^{\mathrm{a}}$ & $0.73 \pm 0.01^{\mathrm{a}}$ & $0.34 \pm 0.00^{\mathrm{a}}$ & $0.24 \pm 0.01^{\mathrm{a}}$ & $0.22 \pm 0.00^{\mathrm{a}}$ & $14.83 \pm 0.44^{\mathrm{a}}$ \\
\hline & 7 & $2.02 \pm 0.01^{\mathrm{b}}$ & $0.96 \pm 0.00^{\mathrm{b}}$ & $0.55 \pm 0.01^{\mathrm{b}}$ & $0.50 \pm 0.00^{\mathrm{b}}$ & $0.33 \pm 0.00^{\mathrm{b}}$ & $18.33 \pm 0.33^{b}$ \\
\hline & 14 & $2.97 \pm 0.01^{\mathrm{c}}$ & $1.48 \pm 0.01^{\mathrm{c}}$ & $0.90 \pm 0.00^{\mathrm{c}}$ & $0.59 \pm 0.01^{\mathrm{c}}$ & $0.25 \pm 0.00^{c}$ & $22.50 \pm 0.29^{c}$ \\
\hline & 21 & $3.98 \pm 0.01^{\mathrm{d}}$ & $2.09 \pm 0.02^{\mathrm{d}}$ & $1.23 \pm 0.01^{\mathrm{d}}$ & $0.66 \pm 0.01^{\mathrm{d}}$ & $0.20 \pm 0.00^{\mathrm{d}}$ & $32.50 \pm 0.29^{d}$ \\
\hline \multirow[t]{4}{*}{ ST2 } & 0 & $1.30 \pm 0.01^{\mathrm{a}}$ & $0.72 \pm 0.01^{\mathrm{a}}$ & $0.35 \pm 0.01^{\mathrm{a}}$ & $0.23 \pm 0.01^{\mathrm{a}}$ & $0.22 \pm 0.01^{\mathrm{a}}$ & $15.17 \pm 0.60^{\mathrm{a}}$ \\
\hline & 7 & $1.84 \pm 0.01^{\mathrm{b}}$ & $0.88 \pm 0.01^{b}$ & $0.54 \pm 0.01^{b}$ & $0.42 \pm 0.00^{\mathrm{b}}$ & $0.29 \pm 0.00^{\mathrm{b}}$ & $20.00 \pm 0.58^{b}$ \\
\hline & 14 & $2.56 \pm 0.01^{\mathrm{c}}$ & $1.32 \pm 0.01^{\mathrm{c}}$ & $0.76 \pm 0.01^{\mathrm{c}}$ & $0.49 \pm 0.00^{c}$ & $0.23 \pm 0.00^{c}$ & $21.83 \pm 0.44^{\mathrm{c}}$ \\
\hline & 21 & $3.25 \pm 0.06^{\mathrm{d}}$ & $1.77 \pm 0.05^{\mathrm{d}}$ & $0.94 \pm 0.00^{\mathrm{d}}$ & $0.54 \pm 0.00^{\mathrm{d}}$ & $0.20 \pm 0.00^{\mathrm{d}}$ & $25.50 \pm 0.29^{d}$ \\
\hline \multirow[t]{4}{*}{ ST3 } & 0 & $1.29 \pm 0.01^{\mathrm{a}}$ & $0.74 \pm 0.00^{\mathrm{a}}$ & $0.34 \pm 0.00^{\mathrm{a}}$ & $0.22 \pm 0.00^{\mathrm{a}}$ & $0.20 \pm 0.00^{\mathrm{a}}$ & $15.50 \pm 0.29^{\mathrm{a}}$ \\
\hline & 7 & $1.52 \pm 0.01^{\mathrm{b}}$ & $0.86 \pm 0.02^{b}$ & $0.36 \pm 0.01^{b}$ & $0.31 \pm 0.01^{\mathrm{b}}$ & $0.25 \pm 0.01^{\mathrm{b}}$ & $16.83 \pm 0.44^{\mathrm{b}}$ \\
\hline & 14 & $1.83 \pm 0.01^{\mathrm{c}}$ & $0.91 \pm 0.01^{\mathrm{b}}$ & $0.54 \pm 0.00^{c}$ & $0.37 \pm 0.00^{c}$ & $0.26 \pm 0.00^{\mathrm{ab}}$ & $19.50 \pm 0.29^{c}$ \\
\hline & 21 & $2.70 \pm 0.04^{\mathrm{d}}$ & $1.56 \pm 0.04^{\mathrm{c}}$ & $0.72 \pm 0.01^{\mathrm{d}}$ & $0.42 \pm 0.00^{\mathrm{d}}$ & $0.19 \pm 0.00^{\mathrm{c}}$ & $22.67 \pm 0.33^{\mathrm{d}}$ \\
\hline
\end{tabular}




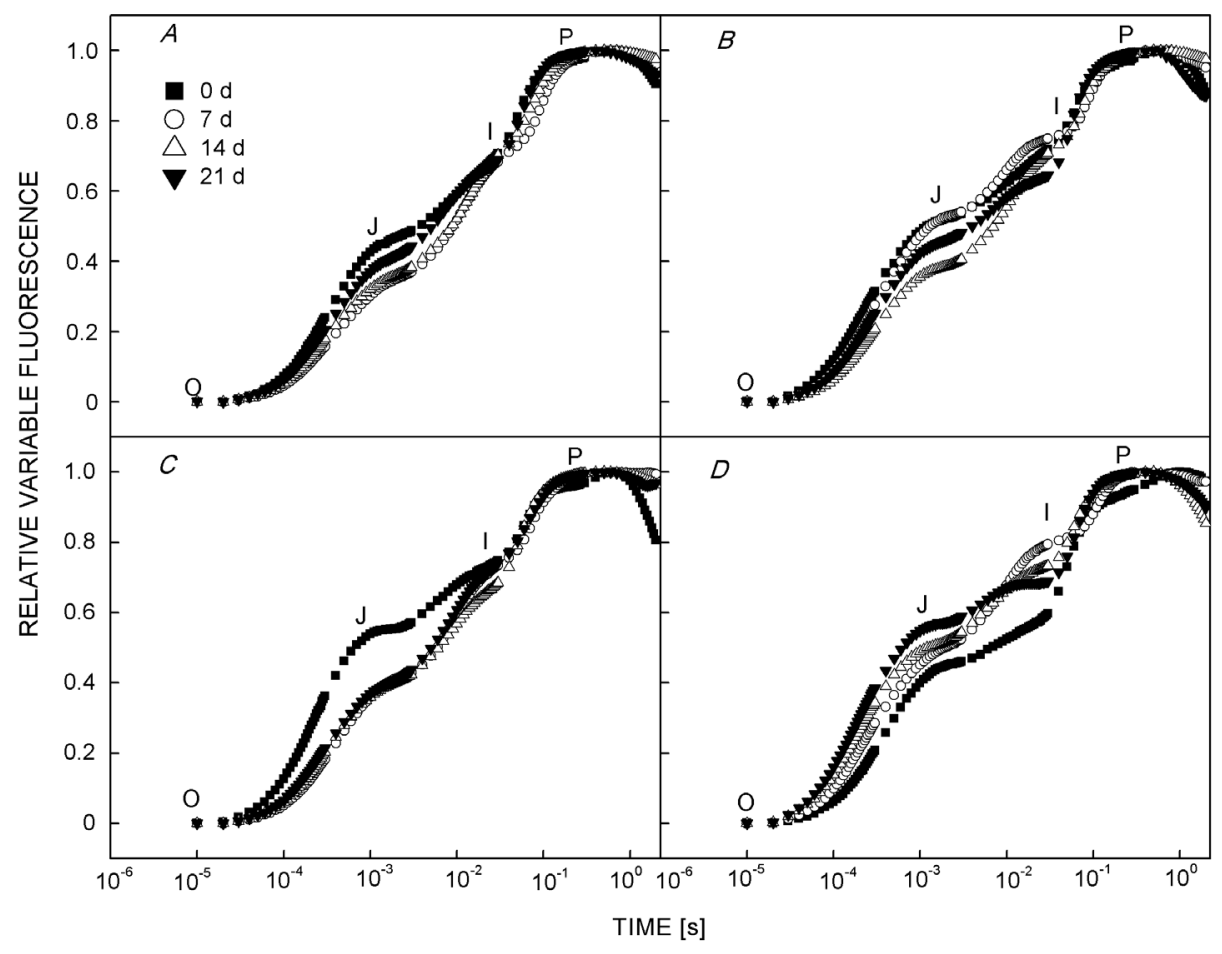

Fig. 2. Fast chlorophyll fluorescence transients (OJIP) of control (CK) $(A)$, and salt-treated: $0.1 \mathrm{~mol} \mathrm{~L}^{-1}(\mathrm{ST} 1)(B), 0.3 \mathrm{~mol} \mathrm{~L}-1$ (ST2) (C), and $0.5 \mathrm{~mol} \mathrm{~L}^{-1}$ (ST3) (D) Lycium ruthenicum Murr. seedlings.

Table 2. Changes of the minimal fluorescence $\left(\mathrm{F}_{0}\right)$, maximum fluorescence $\left(\mathrm{F}_{\mathrm{m}}\right)$, maximum quantum yield of PSII $\left(\mathrm{F}_{\mathrm{v}} / \mathrm{F}_{\mathrm{m}}\right)$, and relative variable fluorescence $\left(\mathrm{V}_{\mathrm{j}}\right)$ of Lycium ruthenicum Murr. seedlings under control (CK), and salt treatments: $0.1 \mathrm{~mol} \mathrm{~L}^{-1}$ (ST1), 0.3 mol $\mathrm{L}^{-1}(\mathrm{ST} 2)$, and $0.5 \mathrm{~mol} \mathrm{~L}^{-1}(\mathrm{ST} 3)$. Data are means $\pm \mathrm{SE}(n=5)$. Different letters indicate significant differences between treatments at $P<0.05$. DAE - days of experiment.

\begin{tabular}{lcllll}
\hline Treatment & DAE & $\mathrm{F}_{0}$ & $\mathrm{~F}_{\mathrm{m}}$ & $\mathrm{F}_{\mathrm{v}} / \mathrm{F}_{\mathrm{m}}$ & $\mathrm{V}_{\mathrm{j}}$ \\
\hline CK & 0 & $192.33 \pm 3.48^{\mathrm{a}}$ & $1455.67 \pm 4.91^{\mathrm{a}}$ & $0.84 \pm 0.00^{\mathrm{a}}$ & $0.39 \pm 0.05^{\mathrm{a}}$ \\
& 7 & $193.80 \pm 6.31^{\mathrm{a}}$ & $1422.80 \pm 22.18^{\mathrm{a}}$ & $0.84 \pm 0.01^{\mathrm{a}}$ & $0.36 \pm 0.04^{\mathrm{a}}$ \\
& 14 & $193.40 \pm 14.46^{\mathrm{a}}$ & $1392.80 \pm 23.81^{\mathrm{a}}$ & $0.81 \pm 0.01^{\mathrm{b}}$ & $0.37 \pm 0.01^{\mathrm{a}}$ \\
& 21 & $199.40 \pm 2.69^{\mathrm{a}}$ & $1422.80 \pm 24.37^{\mathrm{a}}$ & $0.81 \pm 0.01^{\mathrm{b}}$ & $0.39 \pm 0.04^{\mathrm{a}}$ \\
$\mathrm{ST} 1$ & 0 & $190.33 \pm 1.33^{\mathrm{a}}$ & $1434.67 \pm 4.81^{\mathrm{a}}$ & $0.83 \pm 0.01^{\mathrm{a}}$ & $0.37 \pm 0.01^{\mathrm{a}}$ \\
& 7 & $216.40 \pm 14.23^{\mathrm{a}}$ & $1327.60 \pm 37.20^{\mathrm{b}}$ & $0.82 \pm 0.00^{\mathrm{a}}$ & $0.40 \pm 0.06^{\mathrm{a}}$ \\
& 14 & $254.80 \pm 11.91^{\mathrm{b}}$ & $1060.20 \pm 9.35^{\mathrm{c}}$ & $0.81 \pm 0.01^{\mathrm{a}}$ & $0.42 \pm 0.02^{\mathrm{a}}$ \\
& 21 & $275.40 \pm 2.82^{\mathrm{b}}$ & $1028.80 \pm 38.07^{\mathrm{c}}$ & $0.77 \pm 0.02^{\mathrm{b}}$ & $0.43 \pm 0.01^{\mathrm{a}}$ \\
$\mathrm{ST2}$ & 0 & $191.33 \pm 2.85^{\mathrm{a}}$ & $1462.00 \pm 12.86^{\mathrm{a}}$ & $0.84 \pm 0.00^{\mathrm{a}}$ & $0.35 \pm 0.01^{\mathrm{a}}$ \\
& 7 & $219.20 \pm 5.08^{\mathrm{a}}$ & $1325.00 \pm 19.90^{\mathrm{b}}$ & $0.81 \pm 0.00^{\mathrm{a}}$ & $0.43 \pm 0.01^{\mathrm{b}}$ \\
& 14 & $266.20 \pm 15.09^{\mathrm{b}}$ & $1040.40 \pm 10.73^{\mathrm{c}}$ & $0.78 \pm 0.03^{\mathrm{a}}$ & $0.44 \pm 0.01^{\mathrm{ab}}$ \\
$\mathrm{ST} 3$ & 21 & $292.20 \pm 15.23^{\mathrm{b}}$ & $1011.60 \pm 23.64^{\mathrm{c}}$ & $0.70 \pm 0.01^{\mathrm{b}}$ & $0.45 \pm 0.01^{\mathrm{b}}$ \\
& 0 & $190.00 \pm 2.89^{\mathrm{a}}$ & $1459.33 \pm 15.25^{\mathrm{a}}$ & $0.83 \pm 0.01^{\mathrm{a}}$ & $0.39 \pm 0.04^{\mathrm{a}}$ \\
& 7 & $260.60 \pm 15.19^{\mathrm{b}}$ & $1300.00 \pm 58.78^{\mathrm{a}}$ & $0.80 \pm 0.00^{\mathrm{a}}$ & $0.51 \pm 0.03^{\mathrm{b}}$ \\
& 14 & $320.20 \pm 10.38^{\mathrm{c}}$ & $1037.00 \pm 48.89^{\mathrm{b}}$ & $0.68 \pm 0.02^{\mathrm{b}}$ & $0.55 \pm 0.02^{\mathrm{b}}$ \\
& 21 & $369.80 \pm 21.40^{\mathrm{d}}$ & $991.20 \pm 75.78^{\mathrm{b}}$ & $0.63 \pm 0.05^{\mathrm{b}}$ & $0.59 \pm 0.04^{\mathrm{b}}$ \\
\hline
\end{tabular}

stages under CK treatment. Under salt stress, the initial fluorescence $\mathrm{O}$ step did not change compared to that in $\mathrm{CK}$. The O-J, J-I, and I-P stages clearly increased under salt stress treatment compared with that under CK treatment.
However, the highest values of $\mathrm{J}$ and I appeared at 21 and 7 DAE in ST3.

Chl fluorescence transient parameters: $F_{0}$ remained 


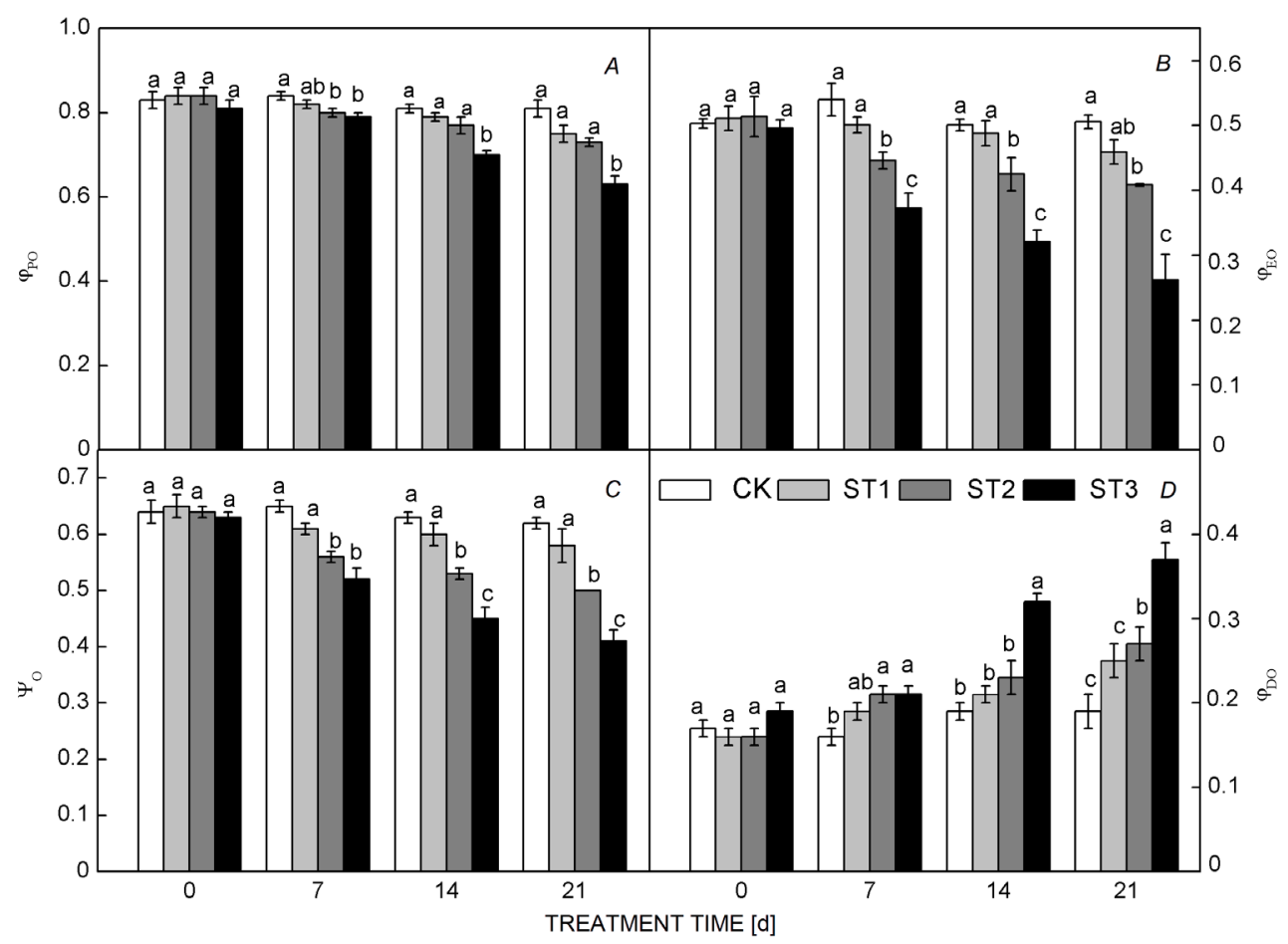

Fig. 3. Maximum quantum yield of primary photochemistry $\left(\varphi_{\mathrm{PO}}\right)(A)$, quantum yield for electron transport $\left(\varphi_{\mathrm{EO}}\right)(B)$, probability that a trapped exciton moves an electron into the trapped electron transport chain beyond $\mathrm{Q}_{\mathrm{A}}\left(\psi_{\mathrm{o}}\right)(C)$, and quantum yield at $\mathrm{t}=0$ for energy dissipation $\left(\varphi_{\mathrm{DO}}\right)(D)$ of control and salt-treated Lycium ruthenicum Murr. seedlings. Data are means $\pm \mathrm{SE}(n=5)$. Different letters indicate significant differences between treatments at $P<0.05$. CK - control, and salt treatments: $0.1 \mathrm{~mol} \mathrm{~L}^{-1}(\mathrm{ST} 1), 0.3 \mathrm{~mol} \mathrm{~L}{ }^{-1}$ (ST2), and $0.5 \mathrm{~mol} \mathrm{~L}^{-1}$ (ST3).

Table 3. Changes of absorption flux per reaction center $(\mathrm{RC})(\mathrm{ABS} / \mathrm{RC})$, dissipated energy flux per $\mathrm{RC}\left(\mathrm{DI}_{0} / \mathrm{RC}\right)$, trapped energy flux per $\mathrm{RC}\left(\mathrm{TR}_{0} / \mathrm{RC}\right)$, electron transport flux per $\mathrm{RC}\left(\mathrm{ET}_{0} / \mathrm{RC}\right)$ of Lycium ruthenicum Murr. seedlings under control (CK), and salt treatments: $0.1 \mathrm{~mol} \mathrm{~L}^{-1}$ (ST1), $0.3 \mathrm{~mol} \mathrm{~L}^{-1}$ (ST2), and $0.5 \mathrm{~mol} \mathrm{~L}^{-1}(\mathrm{ST} 3)$. Data are means $\pm \mathrm{SE}(n=5)$. Different letters indicate significant differences between treatments at $P<0.05$. DAE - days of experiment.

\begin{tabular}{lcllll}
\hline Treatment & DAE & ABS/RC & $\mathrm{DI}_{0} / \mathrm{RC}$ & $\mathrm{TR}_{0} / \mathrm{RC}$ & $\mathrm{ET}_{0} / \mathrm{RC}$ \\
\hline CK & 0 & $2.25 \pm 0.04^{\mathrm{a}}$ & $0.31 \pm 0.01^{\mathrm{a}}$ & $1.65 \pm 0.03^{\mathrm{a}}$ & $0.94 \pm 0.01^{\mathrm{a}}$ \\
& 7 & $2.27 \pm 0.15^{\mathrm{a}}$ & $0.30 \pm 0.03^{\mathrm{a}}$ & $1.65 \pm 0.05^{\mathrm{a}}$ & $0.97 \pm 0.05^{\mathrm{a}}$ \\
& 14 & $2.21 \pm 0.08^{\mathrm{a}}$ & $0.32 \pm 0.02^{\mathrm{a}}$ & $1.66 \pm 0.03^{\mathrm{a}}$ & $0.97 \pm 0.01^{\mathrm{a}}$ \\
& 21 & $2.26 \pm 0.14^{\mathrm{a}}$ & $0.31 \pm 0.02^{\mathrm{a}}$ & $1.63 \pm 0.03^{\mathrm{a}}$ & $0.93 \pm 0.06^{\mathrm{a}}$ \\
ST1 & 0 & $2.22 \pm 0.12^{\mathrm{a}}$ & $0.31 \pm 0.02^{\mathrm{a}}$ & $1.65 \pm 0.09^{\mathrm{a}}$ & $0.94 \pm 0.02^{\mathrm{ab}}$ \\
& 7 & $2.36 \pm 0.09^{\mathrm{ab}}$ & $0.34 \pm 0.01^{\mathrm{a}}$ & $1.70 \pm 0.02^{\mathrm{a}}$ & $0.82 \pm 0.06^{\mathrm{a}}$ \\
& 14 & $2.48 \pm 0.16^{\mathrm{ab}}$ & $0.43 \pm 0.04^{\mathrm{b}}$ & $1.75 \pm 0.11^{\mathrm{a}}$ & $1.03 \pm 0.02^{\mathrm{b}}$ \\
ST2 & 21 & $2.62 \pm 0.05^{\mathrm{b}}$ & $0.48 \pm 0.06^{\mathrm{b}}$ & $1.82 \pm 0.03^{\mathrm{a}}$ & $0.96 \pm 0.07^{\mathrm{b}}$ \\
& 0 & $2.27 \pm 0.15^{\mathrm{a}}$ & $0.31 \pm 0.00^{\mathrm{a}}$ & $1.64 \pm 0.01^{\mathrm{a}}$ & $0.91 \pm 0.02^{\mathrm{a}}$ \\
& 7 & $2.48 \pm 0.07^{\mathrm{a}}$ & $0.48 \pm 0.04^{\mathrm{ab}}$ & $1.72 \pm 0.02^{\mathrm{a}}$ & $0.91 \pm 0.07^{\mathrm{a}}$ \\
ST3 & 14 & $2.66 \pm 0.10^{\mathrm{b}}$ & $0.54 \pm 0.13^{\mathrm{ab}}$ & $1.87 \pm 0.11^{\mathrm{b}}$ & $1.08 \pm 0.05^{\mathrm{b}}$ \\
& 21 & $2.86 \pm 0.05^{\mathrm{b}}$ & $0.73 \pm 0.10^{\mathrm{b}}$ & $1.96 \pm 0.02^{\mathrm{b}}$ & $1.07 \pm 0.03^{\mathrm{b}}$ \\
& 0 & $2.20 \pm 0.03^{\mathrm{a}}$ & $0.30 \pm 0.00^{\mathrm{a}}$ & $1.65 \pm 0.04^{\mathrm{a}}$ & $0.94 \pm 0.01^{\mathrm{a}}$ \\
& 7 & $2.54 \pm 0.10^{\mathrm{a}}$ & $0.53 \pm 0.07^{\mathrm{a}}$ & $1.96 \pm 0.06^{\mathrm{b}}$ & $0.96 \pm 0.06^{\mathrm{b}}$ \\
& 14 & $3.32 \pm 0.14^{\mathrm{b}}$ & $1.07 \pm 0.12^{\mathrm{b}}$ & $2.25 \pm 0.06^{\mathrm{c}}$ & $1.14 \pm 0.02^{\mathrm{c}}$ \\
& 21 & $3.68 \pm 0.30^{\mathrm{b}}$ & $1.40 \pm 0.27^{\mathrm{b}}$ & $2.27 \pm 0.07^{\mathrm{c}}$ & $0.92 \pm 0.06^{\mathrm{c}}$ \\
\hline
\end{tabular}

constant in CK but increased with increasing salinity and stress duration (Table 2). The $\mathrm{F}_{0}$ values in the $\mathrm{CK}$ and salt stress-treated plants were significantly different after
7 DAE. $F_{m}$ decreased in the salt-stressed groups with increasing stress duration. After $7 \mathrm{DAE}$, the $\mathrm{F}_{\mathrm{m}}$ values in the salt-stressed plants were significantly different from 
Table 4. Changes of dissipated energy flux per cross section (CS) $\left(\mathrm{DI}_{0} / \mathrm{CS}_{0}\right)$, trapped energy flux per $\left.\mathrm{CS}_{(\mathrm{TR}} / \mathrm{CS}_{0}\right)$, electron transport flux per CS $\left(\mathrm{ET}_{0} / \mathrm{CS}_{0}\right)$, and amount of active PSII reaction centers per CS ( $\left.\mathrm{RC} / \mathrm{CS}_{0}\right)$ of Lycium ruthenicum Murr. seedlings under control (CK), and salt treatments: $0.1 \mathrm{~mol} \mathrm{~L}^{-1}(\mathrm{ST} 1), 0.3 \mathrm{~mol} \mathrm{~L}^{-1}(\mathrm{ST} 2)$, and $0.5 \mathrm{~mol} \mathrm{~L}^{-1}$ (ST3). Data are means $\pm \mathrm{SE}(n=5)$. Different letters indicate significant differences between treatments at $P<0.05$. DAE - days of experiment.

\begin{tabular}{lcclll}
\hline Treatment & $\mathrm{DAE}$ & $\mathrm{DI}_{0} / \mathrm{CS}_{0}$ & $\mathrm{TR}_{0} / \mathrm{CS}_{0}$ & $\mathrm{ET}_{0} / \mathrm{CS}_{0}$ & $\mathrm{RC} / \mathrm{CS}_{0}$ \\
\hline CK & 0 & $37.29 \pm 2.70^{\mathrm{a}}$ & $182.75 \pm 6.71^{\mathrm{a}}$ & $104.89 \pm 13.13^{\mathrm{a}}$ & $105.36 \pm 8.81^{\mathrm{a}}$ \\
& 7 & $34.05 \pm 3.47^{\mathrm{a}}$ & $183.75 \pm 6.46^{\mathrm{a}}$ & $106.94 \pm 9.62^{\mathrm{a}}$ & $106.77 \pm 6.92^{\mathrm{a}}$ \\
& 14 & $37.22 \pm 2.59^{\mathrm{a}}$ & $186.18 \pm 26.82^{\mathrm{a}}$ & $110.44 \pm 3.98^{\mathrm{a}}$ & $103.42 \pm 10.02^{\mathrm{a}}$ \\
& 21 & $39.64 \pm 2.59^{\mathrm{a}}$ & $187.77 \pm 6.22^{\mathrm{a}}$ & $109.93 \pm 12.81^{\mathrm{a}}$ & $106.61 \pm 2.57^{\mathrm{a}}$ \\
ST1 & 0 & $33.27 \pm 3.57^{\mathrm{a}}$ & $182.15 \pm 2.48^{\mathrm{a}}$ & $108.80 \pm 4.38^{\mathrm{a}}$ & $103.31 \pm 2.53^{\mathrm{a}}$ \\
& 7 & $45.43 \pm 1.40^{\mathrm{a}}$ & $183.77 \pm 3.43^{\mathrm{a}}$ & $89.78 \pm 5.50^{\mathrm{a}}$ & $85.99 \pm 12.05^{\mathrm{a}}$ \\
& 14 & $50.75 \pm 4.38^{\mathrm{a}}$ & $220.05 \pm 8.42^{\mathrm{b}}$ & $132.21 \pm 8.42^{\mathrm{a}}$ & $124.34 \pm 16.97^{\mathrm{a}}$ \\
& 21 & $52.96 \pm 10.81^{\mathrm{a}}$ & $186.91 \pm 6.84^{\mathrm{a}}$ & $95.88 \pm 6.78^{\mathrm{b}}$ & $168.43 \pm 21.21^{\mathrm{b}}$ \\
ST2 & 0 & $33.72 \pm 1.64^{\mathrm{a}}$ & $180.93 \pm 1.16^{\mathrm{a}}$ & $106.52 \pm 9.75^{\mathrm{a}}$ & $103.47 \pm 2.85^{\mathrm{a}}$ \\
& 7 & $50.97 \pm 4.27^{\mathrm{b}}$ & $199.20 \pm 9.59^{\mathrm{a}}$ & $108.10 \pm 6.03^{\mathrm{a}}$ & $120.36 \pm 9.55^{\mathrm{a}}$ \\
& 14 & $64.54 \pm 7.67^{\mathrm{b}}$ & $229.12 \pm 8.88^{\mathrm{b}}$ & $133.77 \pm 8.94^{\mathrm{a}}$ & $141.25 \pm 12.76^{\mathrm{a}}$ \\
$\mathrm{ST} 3$ & 21 & $80.49 \pm 5.47^{\mathrm{c}}$ & $195.25 \pm 13.21^{\mathrm{a}}$ & $115.45 \pm 11.45^{\mathrm{a}}$ & $204.24 \pm 24.12^{\mathrm{b}}$ \\
& 0 & $33.02 \pm 0.68^{\mathrm{a}}$ & $180.98 \pm 5.08^{\mathrm{a}}$ & $103.12 \pm 1.99^{\mathrm{a}}$ & $102.11 \pm 1.40^{\mathrm{a}}$ \\
& 7 & $63.20 \pm 2.57^{\mathrm{b}}$ & $209.63 \pm 11.48^{\mathrm{ab}}$ & $102.51 \pm 9.04^{\mathrm{a}}$ & $104.50 \pm 5.73^{\mathrm{a}}$ \\
& 14 & $109.08 \pm 11.47^{\mathrm{c}}$ & $231.66 \pm 24.23^{\mathrm{b}}$ & $108.78 \pm 8.31^{\mathrm{a}}$ & $106.75 \pm 7.41^{\mathrm{a}}$ \\
& 21 & $133.90 \pm 24.82^{\mathrm{d}}$ & $217.90 \pm 10.49^{\mathrm{ab}}$ & $88.45 \pm 8.30^{\mathrm{a}}$ & $137.67 \pm 13.55^{\mathrm{b}}$ \\
\hline
\end{tabular}

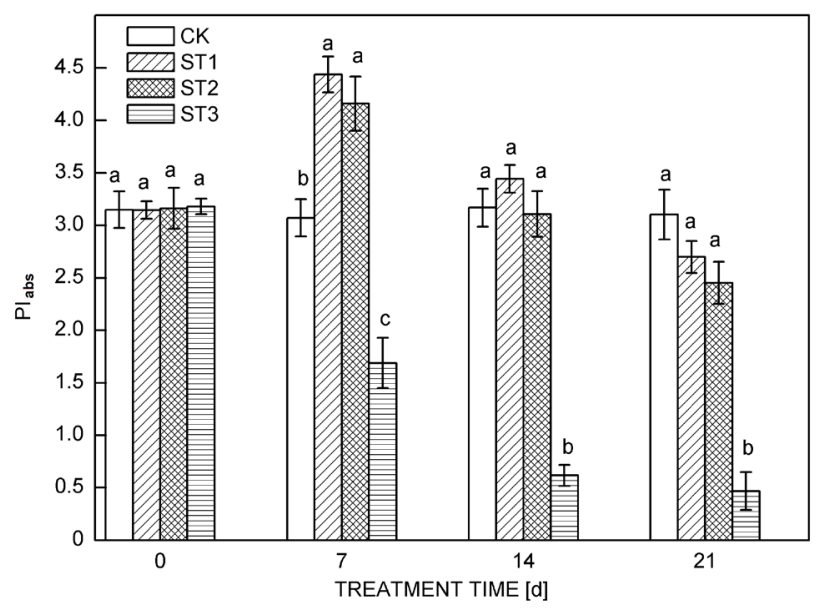

Fig. 4. Performance index on absorption basis $\left(\mathrm{PI}_{\mathrm{abs}}\right)$ of control and salt-treated Lycium ruthenicum Murr. seedlings. Data are means $\pm \mathrm{SE}(n=5)$. Different letters indicate significant differences between treatments at $P<0.05$. CK - control, and salt treatments: $0.1 \mathrm{~mol} \mathrm{~L}^{-1}$ (ST1), $0.3 \mathrm{~mol} \mathrm{~L}^{-1}$ (ST2), and $0.5 \mathrm{~mol}$ $\mathrm{L}^{-1}(\mathrm{ST} 3)$.

that of CK. $\mathrm{F}_{\mathrm{v}} / \mathrm{F}_{\mathrm{m}}$ decreased in salt-stressed groups with increasing salinity and stress duration. At $21 \mathrm{DAE}$, the $\mathrm{F}_{\mathrm{v}} / \mathrm{F}_{\mathrm{m}}$ values of ST3 decreased by $4.9,13.6$, and $22.2 \%$ of the values for CK, ST1, and ST2, respectively. The $V_{j}$ values increased with increased salinity and stress duration and were significantly different from that of $\mathrm{CK}$ after 7 DAE.

Flux ratio parameters: The value of $\varphi_{\mathrm{PO}}$, which is the maximal photochemical efficiency of PSII, decreased with increasing salt concentration and stress duration (Fig. 3A). At 21 DAE, the value in ST3 decreased by $21.8,15.9$, and $13.8 \%$ compared to those of CK, ST1, and ST2, respectively. $\varphi_{\mathrm{EO}}$, the quantum yield for electron transport, decreased with increasing salt concentration and stress duration (Fig. 3B). At 21 DAE, ST3 decreased by 48.2, 43.0, and $35.9 \%$ compared to those of CK, ST1, and ST2, respectively. $\psi_{\mathrm{o}}$ is the probability that a trapped exciton moves an electron into the electron transport chain beyond $\mathrm{Q}_{\mathrm{A}}$. The $\psi_{\mathrm{o}}$ values remained constant in $\mathrm{CK}$ and decreased under salt stress treatment with increasing salt concentration and stress duration. At 21 DAE, $\psi_{0}$ in CK was higher by $7.3,19.1$, and $34.7 \%$ than that of $\mathrm{ST} 1, \mathrm{ST} 2$, and ST3, respectively (Fig. $3 C$ ). $\varphi_{\mathrm{DO}}$ is the maximum primary photochemistry quantum yield. $\varphi_{\text {DO }}$ values increased with increasing salt concentration and stress duration, with the value of ST3 increasing by 92.4, 48.4 , and $37.9 \%$ compared to those of CK, ST1, and ST2, respectively (Fig. $3 D$ ) at $21 \mathrm{DAE}$.

Energy fluxes per reaction center: The values of $\mathrm{ABS} / \mathrm{RC}$, $\mathrm{DI}_{0} / \mathrm{RC}$, and $\mathrm{TR}_{0} / \mathrm{RC}$ increased with increasing salt concentration and stress duration (Table 3). In ST3, the ABS/RC, $\mathrm{DI}_{0} / \mathrm{RC}$, and $\mathrm{TR}_{0} / \mathrm{RC}$ values increased significantly. At $21 \mathrm{DAE}$, the ABS/RC values of ST3 increased by 62.4, 40.4 , and $28.4 \%$ compared to those of CK, ST1, and ST2, respectively. The $\mathrm{DI}_{0} / \mathrm{RC}$ values of $\mathrm{ST} 3$ increased 3.52, 1.94 , and 0.93 times of those of CK, ST1, and ST2, respectively, at $21 \mathrm{DAE}$. $\mathrm{ET}_{0} / \mathrm{RC}$ values showed no significant difference between all treatments and treatment times.

Phenomenological flux per cross section: $\mathrm{DI}_{0} / \mathrm{CS}_{0}$ values increased significantly under salt stress treatment 
(Table 4). In ST3, $\mathrm{DI}_{0} / \mathrm{CS}_{0}$ values increased $0.17,0.59$, 1.39 , and 3.18 times at $0,7,14$, and $21 \mathrm{DAE}$, respectively, compared with those of $\mathrm{CK}$. The $\mathrm{TR}_{0} / \mathrm{CS}_{0}$ values also increased with increasing salt stress concentration. At each salt concentration, the $\mathrm{TR}_{0} / \mathrm{CS}_{0}$ values reached the maximum at $14 \mathrm{DAE}$.

$\mathrm{ET}_{0} / \mathrm{CS}_{0}$ values initially increased and subsequently decreased with increasing salt concentration and stress duration, reaching the maximum value at 14 DAE. The $\mathrm{RC} / \mathrm{CS}_{0}$ values increased with increasing salt concentration and stress duration. In ST2, $\mathrm{RC} / \mathrm{CS}_{0}$ values reached the maximum at 14 to $21 \mathrm{DAE}$.

Photosynthetic performance index: The $\mathrm{PI}_{\mathrm{abs}}$ values remained constant in $\mathrm{CK}$ and decreased gradually with increasing stress duration and salt concentration (Fig. 4). At 21 DAE, the $\mathrm{PI}_{\text {abs }}$ values of ST3 decreased by 85.0, 82.7 , and $81.0 \%$ compared to those of CK, ST1, and ST2, respectively. At $21 \mathrm{DAE}$, the $\mathrm{PI}_{\mathrm{abs}}$ values of CK, ST1, ST2, and ST3 decreased by $1.4,14.2,22.5$, and $85.3 \%$ compared to $0 \mathrm{DAE}$, respectively.

\section{Discussion}

In this study, we found that L. ruthenicum seedlings exhibited strong salt tolerance. The seedling growth and dry mass production of $L$. ruthenicum were insensitive to mild and moderate salt stresses (Fig. 1). Under mild and moderate salt-stress treatments, seedling height, base diameter, crown, leaf mass, stem mass, and root mass increased with increasing salt concentration and reached the maximum in ST2. Mild and moderate salt stresses were beneficial to $L$. ruthenicum seedling growth. Similar results were reported in Sophora japonica L. (Zhang et al. 2002), Acer palmatum (Tang et al. 2015), and Beta vulgaris (Choi et al. 2016). With further increase of the salt concentration in soil, the growth of $L$. ruthenicum seedlings became limited. Under the salt stress treatment, roots are the first organ to experience adverse stress signal and produce corresponding physiological changes. With the increase in salt concentration, the L. ruthenicum seedling showed relatively increased allocation of dry matter to the roots. In addition, root length and R:S gradually increased. Under further stress, the main root length and root dry mass decreased. Similar results were reported in Suaeda glauca (Yi et al. 2011) and Quercus virginiana (Wang et al. 2014). Changes in Chl fluorescence can respond to environmental factors affecting plants. The analysis of a rapid Chl fluorescence kinetic curve under different environmental conditions can facilitate an in-depth understanding of the effect of environmental factors on PSII reaction center, plant photosynthetic mechanism adaptation mechanism of photosynthetic apparatus to environment. Rapid Chl fluorescence induced a kinetic curve, which included $\mathrm{O}, \mathrm{J}$, $\mathrm{I}$, and $\mathrm{P} . \mathrm{V}_{\mathrm{j}}$ change indicated the continuous reduction of the electron acceptor, and the PSII reaction center started closing. Phase $\mathrm{O}$ represents a situation where all molecules of the primary electron acceptor of PSII $\left(\mathrm{Q}_{\mathrm{A}}\right)$ are in the oxidized state $(\mathrm{Xu}$ et al. 2015). During transition from phase $\mathrm{O}$ to phase $\mathrm{J}, \mathrm{Q}_{\mathrm{A}}$ was restored to $\mathrm{Q}_{\mathrm{A}}{ }^{-}$. The $\mathrm{V}_{\mathrm{j}}$ value increased slowly under ST1 and ST2, the results showed that electron transport was blocked at PSII donor and acceptor side. The $V_{j}$ value increased significantly under ST3, which showed that salt stress damaged the electron transport of PSII acceptor side, therefore, the growth of L. ruthenicum seedlings was inhibited at ST3.

This study showed that with the increasing salt concentration, the $\mathrm{F}_{0}$ value of $L$. ruthenicum increased and the $F_{m}$ value gradually decreased. $F_{0}$ value at ST3 was higher than that of CK, ST1, and ST2. The results indicated that the leaves of L. ruthenicum suffered from photoinhibition and PSII was damaged, and PSII excitation energy distribution changed under salt stress. However, L. ruthenicum seedlings improved their heat dissipation to consume excessive excitation energy to adapt under saltstress environment at ST1 and ST2. Under ST3, salt stress decreased electron transfer rate, which caused reversible inactivation of PSII reaction center. Similar results were reported by Li et al. (2013), Yang et al. (2013), and Guo et al. (2016).

Chl fluorescence induction kinetic parameters are the important indexes to examine the extent of stress injury. $\mathrm{ABS} / \mathrm{RC}, \mathrm{DI}_{0} / \mathrm{RC}_{0}, \mathrm{TR}_{0} / \mathrm{RC}_{0}$, and $\mathrm{ET}_{0} / \mathrm{RC}$ values increased significantly under salt stress mainly due to efficient enhancers of single reaction centers. In the process, the light energy of absorption and trapping did not increase the energy of $\mathrm{ET}_{0} / \mathrm{CS}_{0}$ during electron transport in unit area and increased the $\mathrm{DI}_{0} / \mathrm{RC}$ and $\mathrm{DI}_{0} / \mathrm{CS}_{0}$ values. The results showed that the light energy of absorption and trapping ensured electron transport, while heat facilitated the largest dissipation. Similar results were reported in Ginkgo biloba (Wei et al. 2012). The self-protection mechanisms of L. ruthenicum seedlings under salt-stress treatment were as follows: the $\varphi_{\mathrm{PO}}$ value decreased with the increasing salt concentration, whereas $\varphi_{\mathrm{DO}}$ increased gradually. The electron transport capacity of PSII donor side was inhibited under salt stress, and the $\psi_{\mathrm{o}}$ and $\varphi_{\mathrm{EO}}$ values were lower than that of CK. Similar results were reported in Capsicum annuum L. var. grossum (Zhang et al. 2016). $\mathrm{F}_{\mathrm{v}} / \mathrm{F}_{\mathrm{m}}$ can indicate the response of plant to stress. $\mathrm{F}_{\mathrm{v}} / \mathrm{F}_{\mathrm{m}}$ gradually decreased under salt stress. The result showed that L. ruthenicum seedlings were suppressed by light. The $\mathrm{PI}_{\mathrm{abs}}$ is the parameter, which reflects the overall status of photosynthetic apparatus (Stirbet et al. 2018). Under ST3, the $\mathrm{PI}_{\mathrm{abs}}$ value of salt stress-treated plants was significantly lower than that of CK. The results showed that leaf photosynthesis was influenced by severe salt stress.

\section{References}

Arshi A., Ahmad A., Aref I.M., Iqbal M.: Comparative studies on antioxidant enzyme action and ion accumulation in soybean cultivars under salinity stress. - J. Environ. Biol. 33: 9-20, 2012.

Ashraf M., Harris P.J.C.: Photosynthesis under stressful environments: An overview. - Photosynthetica 51: 163-190, 2013.

Azeem A., Wu Y.Y., Xing D.K. et al.: Photosynthetic response of two okra cultivars under salt stress and rewatering. - J. Plant. Interact. 12: 67-77, 2017. 
Bray E.A., Bailey-Serres J., Weretilnyk E.: Responses to abiotic stresses. - In: Buchanan B.B., Gruissem W., Jones R.L. (ed.): Biochemistry and Molecular Biology of Plants. Pp. 11581203. American Society of Plant Physiologists, Rockville 2000.

Choi D.-G., Hwang J.-S., Choi S.-C. et al.: The effect on photosynthesis and osmotic regulation in Beta vulgaris L. var. Flavescens DC. by salt stress. - J. Ecol. Environ. 39: 81-90, 2016.

Forni C., Duca D., Glick B.: Mechanisms of plant response to salt and drought stress and their alteration by rhizobacteria. Plant Soil 410: 335-356, 2017.

George S.J, Manoharan D.K., Li J. et al.: Drought and salt stress in Macrotyloma uniflorum leads to common and specific transcriptomic responses and reveals importance of raffinose family oligosaccharides in stress tolerance. - Gene Reports 10: 7-16, 2018.

Guo Y.Y., Yu H.Y., Kong D.S. et al.: Effects of drought stress on growth and chlorophyll fluorescence of Lycium ruthenicum Murr. seedlings. - Photosynthetica 54: 524-531, 2016.

Han D.H., Li S.J., Wang E.J. et al.: [Effect of exogenous calcium on seed germination and seedling physiological characteristics of Lycium ruthenium.] - China. J. Chin. Mater. Med. 39: 3439, 2014. [In Chinese]

He F.L., Zhao M., Wang J.H. et al.: [Response to drought stresses and drought resistances evaluation of seed germination of four desert vegetation.] - Arid Land Geogr. 34: 100-106, 2011. [In Chinese]

Li G.L., Wu H.X., Sun Y.Q.: Response of chlorophyll fluorescence parameters to drought stress in sugar beet seedlings. - Russ. J. Plant Physl+ 60: 337-342, 2013.

Stirbet A., Lazár D., Kromdijk J., Govindjee: Chlorophyll $a$ fluorescence induction: Can just a one-second measurement be used to quantify abiotic stress responses? - Photosynthetica 56: 86-104, 2018.

Strasser R.J., Srivastava A.: Polyphasic chlorophyll $a$ fluorescence transient in plants and cyanobacteria. - Photochem. Photobiol. 61: $32-42,1995$

Sudhir P., Murthy S.D.S.: Effects of salt stress on basic processes of photosynthesis. - Photosynthetica 42: 481-486, 2004.

Tang L., Li Q.Z., Rong L.P., Li S.S.: Effects of salt stress on the growth and leaf chlorophyll fluorescence in Acer palmatum seedling. - Acta Bot. Boreal. Occident. Sin. 35: 2050-2055, 2015. [In Chinese]

Wang S.F., Hu Y.X., Sun H.Q. et al.: [Effects of salt stress on growth and root development of two oak seedlings.] - Acta Ecol. Sin. 34: 1021-1029, 2014. [In Chinese]
Wei X.D., Chen G.X., Shi D.W. et al.: [Effects of drought on fluorescence characteristics of photosystem II in leaves of Ginkgo biloba.] - Acta Ecol. Sin. 32: 7492-7500, 2012. [In Chinese]

Wei Y.Y., Xu Y.C., Lu P. et al:: Salt stress responsiveness of a wild cotton species (Gossypium klotzschianum) based on transcriptomic analysis. - PLoS ONE 12: doi: 10.1371/ journal.pone.0178313, 2017.

Xu J.Z., Wu X.H., Yang Y.J. et al.: Changes in growth, photosynthesis and chlorophyll fluorescence in the freshwater dinoflagellate Peridinium umbonatum (Peridiniales, Pyrrophyta) in response to different temperatures. - Phycologia 55: 469-477, 2015.

Yang W.Q., Gu M.Y., Kou J.C.: Effect of drought and rewatering on the photosynthesis and chlorophyll fluorescence of Coronilla varia. - Acta Agrestia Sin. 21: 1130-1135, 2013. [In Chinese]

Yi L.P. et al.: [Root system characters in growth and distribution among three littoral halophytes.] - Acta Ecol. Sin. 31: 11951202, 2011. [In Chinese]

Yousuf P.Y., Ahmad A., Ganie A.H. et al.: Antioxidant response and proteomic modulations in Indian mustard grown under salt stress. - Plant Growth Regul. 81: 31-50, 2017.

Zhang C.H., Shen Y.B., Yin W.L.: Effect of salt stress on photosynthesis and growth of four tree species seedlings. Sci. Silvae Sin. 38: 27-31, 2002. [In Chinese]

Zhang L., Zhang G.W., Wang Y.H. et al.: Effect of soil salinity on physiological characteristics of functional leaves of cotton plants. - J. Plant. Res. 126: 293-304, 2013.

Zhang R.H., Li J., Guo S.R., Tezuka, T.: Effects of exogenous putrescine on gas-exchange characteristics and chlorophyll fluorescence of $\mathrm{NaCl}$-stressed cucumber seedlings. Photosynth. Res. 100: 155-162, 2009.

Zhang T.X., Ling Z.J., Lin Y.H.: [Effects of salt stress on seed germination, seedling growth and photosynthetic system II photochemical characteristics of Capsicum annuum L. var. grossum.] - Chin. J. Trop. Crops 37: 1766-1773, 2016. [In Chinese]

Zheng J., Ding C.X., Wang L.S. et al.: Anthocyanins composition and antioxidant activity of wild Lycium ruthenicum Murr. from Qinghai-Tibet Plateau. - Food Chem. 126: 859-865, 2011.

Zhou D.D., Liu D.X., Chen M.M. et al.: Photosynthetic characteristics and chlorophyll fluorescence parameters of Celtis sinensis and Ulmus pumila L. seedling under salt stress. Acta Bot. Boreal. Occident. Sin. 36: 1004-1011, 2016. [In Chinese]

(C) The authors. This is an open access article distributed under the terms of the Creative Commons BY-NC-ND Licence. 